\title{
Worksite Health Service
}

National Cancer Institute

\section{Source}

National Cancer Institute. Worksite Health Service. NCI Thesaurus. Code C15877.

Clinical service at the physical site of employment. 